\section{Crystal and electronic structure of Ruthenium(II) complex}

Julia Koziskova ${ }^{1}$, Jozef Kožíšek ${ }^{1}$,

1. Institute of Physical Chemistry and Chemical Physics, Slovak University of Technology in Bratislava, Bratislava, Slovakia

email: julia.koziskova@stuba.sk

The crystal structure of Ruthenium(II) complex, $\mathrm{C}_{24} \mathrm{H}_{26} \mathrm{Cl}$ $\mathrm{N}_{2} \mathrm{O}_{4} \mathrm{Ru} \cdot \mathrm{PF}_{6}$, has been determined and analyzed in terms of connectivity and packing patterns. The compound crystallizes in the monoclinic crystal system in the space group $P 2_{1} / c$. with one cation and one anion in the asymmetric unit [1].

Biological effects of ruthenium complexes and their potential use as metal-based drugs, especially as anticancer agents are one of the leading area. Excluding widespread use of platinum-based chemotherapeutics, ruthenium-based complexes are among the most promising alternatives.

Data collection was performed on a Stoe STADIVARI diffractometer with a Dectris Pilatus $300 \mathrm{~K}$ detector and with an Incoatec $\mathrm{I} \mu \mathrm{S} \mathrm{Ag}$ microfocus source $(\mathrm{Ag}-\mathrm{K} \alpha, \lambda=0.56083 \AA)$ at $100 \mathrm{~K}$ using a nitrogen gas open-flow cooler Cobra from Oxford Cryosystems. Data reduction was processed using X-Area [2]. For numerical absorption corrections a crystal-shape model with 10 faces was employed. An average redundancy of 18.28 gives $\mathrm{R}_{\text {int }}$ of $3.18 \%$. A Hirshfeld surface analysis was carried out and two-dimensional (2D) fingerprint plots [3] were generated to visualize the intermolecular interactions and to provide quantitative data for their relative contributions. Direction cosines were applied for the anisotropic secondary extinction correction. The results of multipole refinement were performed on $\mathrm{F}^{2}$ using XD suite of programs. After the multipole refinement a topological analysis of the charge density was done. In addition, these results were compared with the electrostatic potential from Hirshfeld surfaces.

\section{Acknowledgement}

This work has been carried out with the support of the Ministry of Education, Science, Research and Sport of the Slovak Republic within the Research and Development Operational Programme for the project "University Science Park of STU Bratislava”, ITMS 26240220084, co-funded by the European Regional Development Fund and also by support of the Research and Development Agency under the contract No. APVV-15-0079 and Scientific Grant Agency of the Slovak Republic VEGA (Project Nos. 1/0871/16 and 1/0598/14).

References:

[1] Katja Traven, Iztok Turel, Julia Koziskova and Jozef Kozisek, Concomitant Polymorphism in Organometallic Ruthenium(II) Complex with N,N-donor Ligand, Acta Cryst. C, Submitted. [2] STOE \& Cie GmbH (2016). X-Area 1.76, software package for collecting single-crystal data on STOE area-detector diffractometers, for image processing, scaling reflection intensities and for outlier rejection; Darmstadt, Germany.

[3] Turner, M. J., McKinnon, J. J., Wolff, S. K., Grimwood, D.

J., Spackman, P. R., Jayatilaka, D. \& Spackman, M. A. (2017).

CrystalExplorer17. University of Western Australia. http://

hirshfeldsurface.net

Keywords: ruthenium(II) complex, electrostatic potential, charge density
MS22- Molecular structure and chemical properties: chemistry meets charge density

Chairs: Dr. Vladimir Stilinovic, Dr. Jacob Overgaard

\section{MS22-P01}

\section{HAR and TAAM eefinements of model crystal structures using $\mathrm{CuKa}$ and MoKa $\mathrm{X}$-ray diffraction data}

Monika Wanat ${ }^{1}{ }^{2}$ Maura Malinska ${ }^{2}$, Krzysztof Wozniak ${ }^{2}$

1. College of Inter-Faculty Individual Studies in Mathematics and Natural Sciences (MISMaP), University of Warsaw

2. Biological and Chemical Research Centre, Chemistry Department, University of Warsaw, Warsaw, Poland

email: mwanat@uw.edu.pl

Commonly, the Independent Atom Model (IAM) of electron density is used in the case of routine X-ray data analysis. However, this model does not give a quantitative description of electron density distribution because atoms are assumed to be neutral and spherical. A far better model that allows for modelling of deformation of spherical charge density was introduced by Hansen and Coppens ${ }^{1}$ and is called a pseudoatom model of electron density. Application of this kind of model requires an excellent quality crystals and high resolution XRD data. Quite often, this is difficult to be fulfilled. Therefore, new methods have been developed that enable reconstruction of electron density i.e. Hirshfeld Atom Refinement (HAR) ${ }^{2}$ or Transferable Aspherical Atom Model (TAAM) ${ }^{3}$.

Validation of application of HAR and TAAM methods for low-resolution data will be presented. For three compounds following refinements of $\mathrm{CuK} \alpha$ and $\mathrm{MoK} \alpha$ data will be presented: IAM, TAAM (isotropic $\mathrm{H}$ atoms), TAAM (using Shade), HAR (isotropic H atoms), HAR (anisotropic $\mathrm{H}$ atoms), HAR (using Shade). Additionally, the multipole model and higher order of TAAM and HAR refinements will be presented for MoK $\alpha$ X-ray diffraction data. Analysis of geometry, ADPs (Fig. 1), fractal dimension plots and residual density maps will be shown in comparison with neutron diffraction data.

Fig.1 PEANUT representations of the difference between $A D P S$ obtained from neutron data refinement and HAR, TAAM refinements for $M o K \alpha$ and $C u K \alpha$ data. Root mean square difference scale of 2 was used.
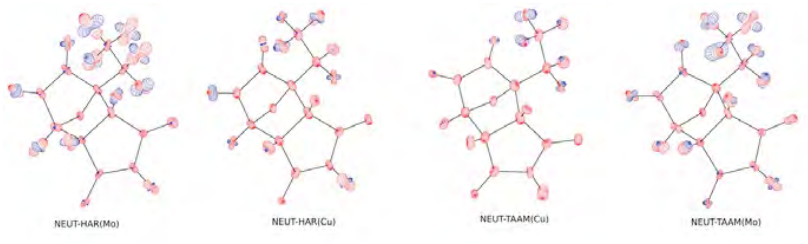
References:

[1] Hansen, N. K.; Coppens, P. (1978). Acta Crystallogr. A. 34 (6). 909-921.

[2]Capelli, S. C.; Bürgi, H.-B.; Dittrich, B.; Grabowsky, S.; Jayatilaka, D. (2014). IUCrJ. 1 (5). 361-379.

[3] Jarzembska, K. N.; Dominiak, P. M. (2012).. Acta Crystallogr. A. 68 (1). 139-147.

Keywords: Hirshfeld Atom Refinement, Transferable Aspherical Atom Model, charge density

\section{MS22-P02}

\section{Experimental charge density for $\sigma$ hole interactions}

Ulli Englert ${ }^{1}$, Ruimin Wang 2 , Daniel Hartnick², Shannon Potts ${ }^{2}$, Irmgard Kalf ${ }^{2}$

1. Inorganic Chemistry, RWTH Aachen University, Aachen, Germany

2. RWTH Aachen, Aachen, Germany

email: ullrich.englert@ac.rwth-aachen.de

Halogen bonds and $\sigma$ hole interactions do not only play an important role in crystal engineering but are also relevant for understanding chemical reactivity.

3,3-dimethyl-1-(trifluoromethyl)-1,3-dihydro-1 $\lambda^{3}, 2$-benziodoxole, 1, commonly known as "Togni reagent" [1], is used for the electrophilic transfer of the trifluoromethyl group by reductive elimination. The $\sigma$ hole associated with the hypervalent iodine atom is essential for this reactivity. [2] Based on high resolution X-ray diffraction, we have determined the experimental electron density for $\mathbf{1}$. In the crystal, it interacts with a neighbouring molecule via $\mathrm{O} \cdots \mathrm{I}$ contacts of 2.9809(6) $\AA$. We compare 1 with two cocrystals 2 and 3 (see Scheme) in terms of electrostatic potential and QTAIM properties in the bond critical point (bcp) for the $\sigma$ hole interaction. In $\mathbf{2}$, tetrafluorodiiodobenzene (TFDIB) interacts with two molecules of dimethylaminopyridine (DMAP); the N $\cdots$ I halogen bond is as short as 2.6622(4) $\AA$. With respect to its electronic properties, it resembles a coordinative bond [3]. 3 consists of infinite chains in which diaminobicyclooctane (DABCO) and TFDIB alternate; the $\mathrm{N} \cdots$ I contacts are significantly longer but still much shorter than $\mathrm{O} \cdots \mathrm{I}$ in the case of the Togni reagent. The bcps for the halogen bonds in $\mathbf{3}$ fall in the range of electron depletion associated with the $\sigma$ hole on iodine; consequently, the electron density in the bcps is surprisingly small.

In conclusion, the three examples documented in this contribution underline the very wide range of $\sigma$ hole interactions.

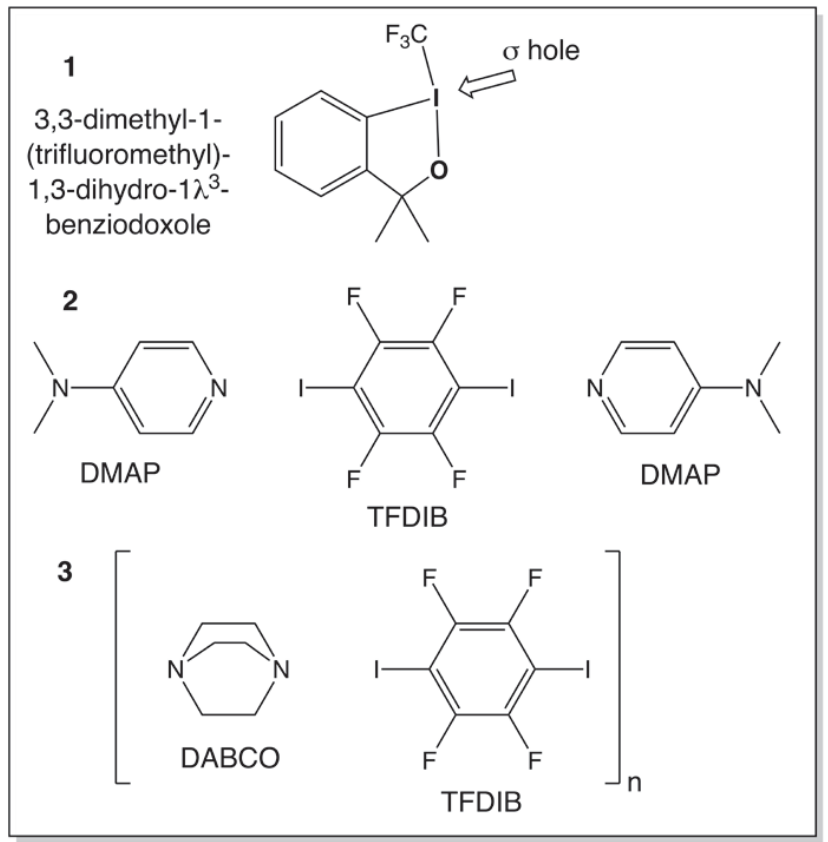

